\title{
Factores psicológicos relacionados con la intencionalidad de abandonar las enseñanzas profesionales de rendimiento musical
}

\author{
Psychological factors related to intent to leave the \\ musical teaching of performance professional
}

\section{Fatores psicológicos relacionados com a intenção de deixar o ensino musical de desempenho}

\author{
Ana García-Dantas ${ }^{1}$, Juan González y Francisco González ${ }^{3}$ \\ 1 Universidad de Sevilla, 2 Universidad de Murcia, 3 Conservatorio de Música de Jaén
}

\begin{abstract}
Resumen: Se presenta aquí un estudio con el objetivo de conocer los factores demográficos y psicológicos que mayor influencia presentan en el cese de las enseńanzas profesionales de música. Una muestra de 113 estudiantes (54 mujeres y 59 varones, con edades comprendidas entre 12 y 45 ańos) perteneciente a un conservatorio contestó el cuestionario MMEPC (García-Dantas y Caracuel, 2011). Los resultados mostraron diferencias significativas entre chicos y chicas puntuando las mujeres menores niveles de competencia percibida y mayores niveles de activación que los hombres. La motivación intrínseca, la competencia percibida y el estrés eran los factores psicológicos que mayor impacto ejercían en la decisión de abandonar. Se espera que las intervenciones psicológicas y formativas a) reduzcan los factores de riesgo que conducen a un abandono desadaptativo $y, b)$ potencie aquellos que promuevan una mayor adherencia y disfrute en el aprendizaje de la música. Palabras clave: abandono, adherencia, músicos, conservatorio, MMEPC.

Abstact: It's presented a study to acknowledge psychological and demographic factors that influence on the professional learning abandon of the music. A sample of 113 students $(54$ females and 59 males, ranged 12 to 45 years old) from a music conservatoire answered the MMEPC questionnaire (García-Dantas \& Caracuel, 2011). Results showed significant differences among females and males, thus women presented less perceived competence and higher activation levels than men. Intrinsic motivation,
\end{abstract}

perceive competence and stress exert the greater impact on the dropping out intention. Psychological and educational interventions are expected to a) reduce the studied risk factors for a desadaptative abandon, b) enhance those factors which promote a greater adherence and enjoyment the learning of the music.

Key words: dropping out, adherence, music, conservatoire, MMEPC.

Resumo: É apresentado um estudo para reconhecer os fatores psicológicos e demográficos que influenciam na aprendizagem profissional abandonar a música. Uma amostra de 113 alunos ( 54 do sexo feminino e 59 do sexo masculino, variou 12 a 45 anos) a partir de um conservatório de música responderam ao questionário MMEPC (García-Dantas \& Caracuel de 2011). Os resultados mostraram diferenças significativas entre mulheres e homens, assim, as mulheres apresentaram menor competência percebida e níveis de ativação mais elevadas do que os homens. A motivação intrínseca, perceber a competência e estresse exercer o maior impacto sobre a intençáo abandono. As intervençóes psicológicas e educacionais são esperados para a) reduzir os fatores de risco estudados por um desadaptative abandono, b) melhorar os fatores que promovem uma maior aderência e diversão a aprendizagem da música.

Palabra chave: abandono, adesão, música, conservatório, MMEPC.

\section{Introducción}

Abandonar o interrumpir una actividad no siempre debe ser entendida de forma negativa. De este modo, el hecho de tener la intención de dejar el conservatorio no tiene por qué implicar de forma directa estados psicológicos desadaptativos. Este deseo podría deberse simplemente a la existencia de otras alternativas más reforzadoras e incompatibles con las exigencias del conservatorio.

No obstante, analizar los factores que influyen en el aban-

Dirección para correspondencia [Correspodence address]: Dr. Juan González Hernández. Dpto. Personalidad, Evaluación y Tratamientos Psicológicos. Facultad de Psicología. Universidad de Murcia. Campus de Espinardo, s/n. 30100. Espinardo (Murcia).E-mail. jgonzalez@um.es dono de una actividad de rendimiento como es la música o la danza es relevante porque estudios previos en deporte han mostrado que, en ocasiones, las transiciones que muchas personas atraviesan son difíciles, y van acompañadas de estados emocionales negativos que pueden resultar en el despliegue de estrategias de afrontamiento inadecuadas (González y Torregrosa, 2009).

Comparado con la psicología aplicada en el ámbito del deporte, que cuenta con numerosos estudios acerca de las variables que influyen en la adherencia y en el abandono de los deportistas (Carlin, Salguero, Márquez y Garcés, 2009; Guillén y Bara, 2009), existen escasas investigaciones sobre este tema en el terreno de la interpretación artística. 
De esta forma, no se han encontrado datos en la literatura que muestren las peculiaridades que presenta este colectivo en comparación con los bailarines y deportistas. Hasta ahora no se sabe qué factores psicológicos o personales inciden en la decisión de abandonar del alumnado profesional de música de los conservatorios; si se trata por conflictos de intereses o tal vez por el curso de determinadas variables, por una indefinición de objetivos o por falta de habilidades de perseverancia; así como tampoco se conoce el porcentaje real de abandono que existe en estas enseñanzas.

García-Dantas y Caracuel (2011) señalan que analizar los motivos que influían en el abandono previo a que este abandono tuviera lugar, podría de alguna forma dilucidar la existencia de ciertas tendencias ante estas situaciones. Así por ejemplo, sería interesante conocer si existen diferencias de género, de edad, de especialidad o incluso de curso en algunas variables psicológicas. Y también, analizar qué variable psicológica está más relacionada con el abandono para que el profesorado pueda trabajar sobre ellas antes de que el alumno o alumna decida abandonar.

Literatura previa en el campo de la psicología aplicada al ámbito musical, ha mostrado de forma recurrente que existen diferencias de género principalmente en dos variables: competencia percibida y ansiedad o nivel de activación (Wehr-Flowers, 2006). Aunque Wehr-Flowers (2006) señala que en estudios anteriores no se encontraron diferencias significativas entre las habilidades reales y el género. North, Colley y Hargreaves (2003) destacan cómo la tarea de componer en música era percibida como una actividad predominantemente masculina entre los estudiantes.

Otros estudios relacionados han mostrado como las chicas tienden a creer menos en sus propias capacidades y suelen tener mayores niveles de activación o ansiedad estado al tocar en solitario (Solmon, Lee y Belcher, 2003; Tavani y Losh, 2003; Vermeer, Boekaerts y Seegers, 2000). Por tanto, parece que existe una tendencia diferente de infravaloración y ansiedad en las chicas en comparación con los chicos.

En este sentido, Wehr-Flowers (2006) en base a una muestra de 137 músicos ( 83 chicos y 54 chicas) analizó si existían diferencias de género en la confianza y ansiedad que ellos sentían cuando tocaban. Los resultados revelaron que las mujeres estaban menos dispuestas a improvisar que los chicos quienes mostraban significativamente mayor confianza en todos los ítems de la escala que ellas. De esta forma indicando que las mujeres presentaban mayores niveles de como competencia percibida que sus compañeros. Del mismo modo, el cuestionario de ansiedad reflejaba diferencias de género, por la cual las chicas presentaban significativamente mayores de ansiedad estado que los chicos cuando tenían que tocar en solitario o improvisar.

Con la intención de conocer si existían también diferencias de género y curso en los conservatorios de danza, Gar-
cía-Dantas y Caracuel (2011) analizaron una muestra de 119 estudiantes de $1^{\circ}$ a $3^{\circ}$ de todas las especialidades de grado profesional. En contraposición con los estudios realizados en música, no se encontraron diferencias de género en la confianza que presentan al bailar ni en el nivel de activación.

Sin embargo se observó que el curso correlacionaba de forma significativa con la presión y de forma decreciente con el clima de clase. Así pues, conforme se avanza de curso la presión aumentaba progresivamente, mostrando significativamente mayor presión aquellas personas que estaban en $3^{\circ}$ de Grado Profesional que las que estaban en $1^{\circ}$. De forma opuesta, se encontró como el clima de clase en las aulas de danza desciende a lo largo de los años, afirmando tener los cursos inferiores mejores relaciones sociales que los mayores.

De la misma forma, la competencia percibida o la valoración que se otorgaban a ellos/as mismos/as a la hora de bailar (García-Dantas y Caracuel, 2011), variable que no se relaciona significativamente con la ansiedad generada por la falta de tiempo para estudiar y con la motivación básica.

Por otro lugar, otros estudios afirman que el abandono en danza se relaciona de forma significativa con la motivación básica y cotidiana, con el apoyo percibido de los profesores y con el nivel de competencia percibida. En todas ellas la relación con la probabilidad de abandono era inversa (GarcíaDantas, Caracuel y Peñaloza, 2013).

Si se generalizan estos resultados de danza a la población de música, es esperable que si se incrementa la motivación, el apoyo percibido del profesorado y la percepción que ellos mismos tienen al tocar, con mucha probabilidad la intención de abandonar se reducirá (García-Dantas et al., 2013). Actualmente la tasa de intención de abandono, se observa cómo un $15 \%$ de personas estaban pensando en abandonar en ese momento; casi un $40 \%$ del total lo habían pensado alguna vez a lo largo de los años, y sólo un 45,4\% no se lo había planteado nunca.

En otro estudio (Caracuel, García-Dantas y González, 2011), se encuentran similitudes y diferencias entre 233 alumnos de conservatorio de música y danza, mostró que ambos colectivos otorgaban similares valores a la competencia percibida, apoyo de los padres, estatus social -nivel artístico percibido en comparación con el grupo clase- y clima de grupo. Sin embargo, mientras que los bailarines presentaron mayor motivación básica y cotidiana por asistir a clase, también mostraron mayor nivel de activación, estrés y falta de tiempo para estudiar que los músicos. En ese mismo trabajo, mientras que los músicos afirmaron tener mayor apoyo de los profesores, menor estrés y falta de tiempo para estudiar, presentaron menor motivación que los bailarines, aunque no desarrollan ideas de abandono.

Por tanto, los objetivos del presente estudio son: a) conocer si existen diferencias de género, curso y edad en algunas de las variables estudiadas, b) mostrar las variables que mayor 
influencia presentan en la intención de abandonar y, c) presentar el porcentaje real de intención de abandono que existe actualmente en el alumnado de $1^{\circ}$ a $3^{\circ}$ de grado profesional de un Conservatorio de Música de Andalucía.

\section{Método}

\section{Participantes}

En este estudio participaron 113 estudiantes (54 mujeres y 59 varones, de entre 12 y 45 ańos), del Conservatorio Profesional de Música de Jaén. Se distribuían entre los cursos, primero, segundo y tercero de Grado Medio de las 13 especialidades existentes: chelo $(0,9 \%$ de músicos), saxofón $(11,4 \%)$, clarinete $(12,3 \%)$, fagot $(5,3 \%)$, flauta travesera $(9,6 \%)$, guitarra $(3,5 \%)$, oboe $(7 \%)$, percusión $(5,3 \%)$, piano $(7,9 \%)$, trombón $(12,3 \%)$, trompa $(5,3 \%)$, trompeta $(16,7 \%)$ y tuba $(1,8 \%)$ durante el curso 2009-2010. El porcentaje de participantes distribuidos según las categorías antes señaladas aparece en la Tabla 1.

Tabla 1. Porcentaje de estudiantes en función del género, edad y curso de Grado Profesional.

\begin{tabular}{llllllllll}
\hline Género & \multicolumn{3}{c}{ Edad } & \multicolumn{5}{c}{ Curso } \\
\hline Chicos & Chicas & $12-14$ & $15-17$ & $18-20$ & $21-23$ & $24-45$ & $1^{\circ}$ & $2^{\circ}$ & $3^{\circ}$ \\
52,6 & 47,4 & 64,8 & 21 & 10,6 & 1,8 & 1,8 & 25,4 & 50 & 24,6 \\
\hline
\end{tabular}

\section{Procedimiento}

Se accedió a la muestra tras la aprobación por parte del equipo directivo a realizar la investigación y la posterior conformidad del profesorado en cuestión. A pesar de que se le explicó que la tarea era de carácter voluntaria, el 100\% del alumnado decidió participar en ella tras firmar los correspondientes consentimientos. Del mismo modo, se debe tener en cuenta que todos los estudiantes llevaran en el conservatorio el mismo número de años. En la aplicación, con un sistema ciego, en el que los profesores son los encargados de administrar los cuestionarios, pero sin conocer realmente los objetivos que se pretenden para no sesgar las respuestas de sus alumnos.

\section{Instrumentos}

Para la recogida de información, se ha diseñado un cuestionario ad hoc en el que se reflejan las diferentes variables sociodemográficas, además, se ha adaptado el cuestionario MMEPC (García-Dantas y Caracuel, 2011) originalmente diseñado para bailarines de Conservatorios de danza, con el fin de evaluar los motivos principales que afectan al mantenimiento y al abandono de la Música. Dicho cuestionario se apoya en autores como Martens, Vealey y Burton (1990) y Mérida y Caracuel (2001) o Scanlan (2006), entre otros. Por las escalas que incluye se le denominó MMEPC, de acuerdo con las iniciales de los cinco factores que se diferenciaron tras su análisis factorial y que son los siguientes: Motivación intrínseca (básica y cotidiana), Motivación extrínseca (apoyo social de padres y profesores), Ejecución (competencia y nivel de activación), Presión (estrés y estudios) y Clima social (ambiente de clase).

El cuestionario consta de 24 ítems que se responden en una escala tipo Likert de 1 a 5, según el grado de acuerdo con cada uno de ellos. Además, cuenta con una escala gradual de 1 a 10 que pregunta por la percepción que la persona tiene de su habilidad o competencia en comparación con el resto de su clase (estatus social). De esta forma se contesta a la pregunta de en que posición te encuentras tú comparado con las demás personas de tu clase. Por último, la variable intención de abandonar contempla tanto el pasado "Alguna vez has pensado en abandonar", como el presente "Actualmente estoy pensando en abandonar". Por tanto, si una persona pensó en abandonar en el pasado y actualmente también tiene intención de abandonar, presentará más probabilidad de abandono que aquella que no lo ha pensado nunca o incluso que lo pensó en un momento puntual del pasado.

\section{Análisis de datos}

El análisis de los datos se llevó a cabo mediante el programa estadístico SPSS, en su versión 19. 0; y además de la valoración descriptiva llevada a cabo, se realiza estudio correlacional de variables sociodemográficas y psicológicas.

\section{Resultados}

Comprobando si existen diferencias estadísticamente significativas en función del género con alguna de las variables psicológicas analizadas, encontramos como las chicos puntúan significativamente más alto en estatus social -percepción del propio rendimiento en comparación con su grupo- que los chicas $\left(\mathrm{t}=2.08, \mathrm{p}=0.04, \mathrm{r}^{2}=0.03\right)$.

Las chicas sufren mayores niveles de activación que los varones, o dicho de otro modo, sufren mayor ansiedad antes de interpretar en solitario, $\left(\mathrm{t}=3.53, \mathrm{p} \leq .01, \mathrm{r}^{2}=.09\right)$. Aunque no se encontraron diferencias estadísticamente significativas en función del curso, sí que se observan ciertas variaciones en 
función de la edad de los participantes. En este sentido, tal y come se observa en la Tabla 2, la edad presenta relación con el estrés, la sensación de falta de tiempo para estudiar materias escolares y la competencia percibida. Por tanto, a mayor edad, el alumnado sufre una mayor tasa de estrés, mayor dificultad en compaginar la música con los estudios del instituto universidad y lo que parece más sorprendente, su competencia percibida decrece significativamente.

Tabla 2. Correlación entre la edad y la variable estrés, estudios y competencia percibida.

\begin{tabular}{llll}
\hline & Estrés & Estudios & Competencia percibida \\
\hline Edad (Person) & $.273^{* *}$ & $.266^{* *}$ & $-.337^{* *}$ \\
\hline Nota: estudios: falta de tiempo para dedicarle a los estudios académicos. \\
${ }^{* *}$ Correlación significativa al .01 (bilateral)
\end{tabular}

En la Tabla 3 se muestra el porcentaje de personas que en el momento de la administración de los cuestionarios deseaban abandonar, aquellas que nunca se lo han planteado y las que desearon hacerlo en el pasado y no actualmente.

Tabla 3. Porcentaje de personas que no desean abandonar, que lo desearon en el pasado y que se lo plantean actualmente

\begin{tabular}{ll}
\hline Situación & Participantes \\
\hline No abandono & $35,1 \%$ \\
Abandono Pasado & $46,5 \%$ \\
Abandono Actual & $17,6 \%$ \\
\hline
\end{tabular}

La Tabla 4 muestra la relación existente entre la probabilidad de abandonar (intención de abandono) con el resto de variables. Tal y como puede observarse, de forma inversamente proporcional, la motivación básica $(\mathrm{p}=-.216)$, la motivación cotidiana $(\mathrm{p}=-.417)$ y la competencia percibida $(\mathrm{p}=-.353)$ y de forma directamente proporcional el estrés $(\mathrm{p}=.181$ ), están estrechamente relacionados con el deseo de dejar el conservatorio.

Tabla 4. Correlaciones entre las escalas estudiadas y la probabilidad de abandonar el Conservatorio

\begin{tabular}{llllllllll}
\hline & Motiv. básica & Mot. cotid. & Prof. & Padr. & Comp. & Nivel Activ. & Estrés & Estud. & Clima \\
\hline Ideas de abandono & $-.216^{* *}$ & $-.417^{* *}$ & -.184 & -.008 & $-.353^{* *}$ & .181 & $.205^{*}$ & .088 & -.160 \\
\hline${ }^{* *}$ Correlación significativa al nivel 0.01 (bilateral) \\
${ }^{*}$ Correlación significativa al nivel 0.05 (bilateral)
\end{tabular}

\section{Discusión}

En primer lugar cabe mencionar las diferencias de género encontradas en algunas variables. Por ejemplo, se observa cómo en el terreno del aprendizaje musical las mujeres puntúan menos en estatus social que los varones. Así, cuando se le pregunta por el lugar que ellos creen que ocupan de 0 a 10 con respecto a los compańeros de su clase, encontramos que las mujeres tienden a valorarse de forma más negativa que los hombres.

De entre todas las variables en las que se podrían haber encontrado diferencias significativas, en música parece que la variable estatus social es la que presenta mayores discrepancias por sexo, datos que se ajustan a los estudios de Wehr-Flowers (2006). En contraste, en el estudio de GarcíaDantas y Caracuel (2011), llevado a cabo en el Conservatorio de Danza de Sevilla, no se apreciaron diferencias en dicha variable, aunque sí en presión. De estos resultados se puede deducir que a pesar de que la danza y la música se consideran dos disciplinas de la interpretación artística, hay factores diferenciales que promueven incluso que chicos y chicas experimenten aspectos psicológicos de forma distinta (Caracuel, García-Dantas y González, 2011).

Cabe plantearse si en algunos casos es posible que se dé un trato diferencial por género, causado por roles tradicionales acerca del uso de determinados instrumentos o por preconcepciones acerca de que la música es una actividad predominantemente masculina (Wehr-Flowers, 2006). Es justo que el profesorado se pregunte cuáles pueden ser los motivos que promuevan esa distinción y si su papel en el aula puede compensar de alguna forma la carencia en una variable tan importante, en el caso de las chicas, como es la valoración que se otorgan a ellas mismas en comparación con su grupo de clase (Bandura, 1997).

Muy relacionado con lo anterior se debe reflexionar acerca de los motivos que llevan a que las chicas sientan más temor a actuar en solitario que los varones. Cuando las chicas del estudio se exponen ante una audición, a un examen o simplemente a la interpretación delante del profesorado, aseguran en un alto porcentaje sufrir mayor tensión y ansiedad que los chicos. Esta variable denominada aquí nivel de activación tiene mucho que ver con el estatus social. De esta forma, si las chicas de la clase tienen una pobre creencia en la interpretación que van a realizar, es lógico que sufran mayores niveles de tensión previos a la actuación (López De La Llave y PérezLlantada, 2006).

Un nivel de activación alto entre las chicas es esperable debido a la existencia de estudios previos que han encontrado datos similares (Wehr-Flowers, 2006). Investigaciones en el ámbito del deporte, ámbito en el que también se plantean 
situaciones de aprendizaje de destrezas y exposición permanente al rendimiento, han mostrado una estrecha relación inversa entre competencia percibida y ansiedad estado o nivel de activación (Marquez, Jerome, Mcauley, Snook, y Canaklisova, 2002). En danza esta relación negativa también se ha encontrado en diferentes investigaciones (Sanna, Nordin-Bates, Cummin, Aways y Sharp, 2011; Walker y Nordin-Bates, 2010). Los datos del presente estudio indican que en música ocurre algo similar.

El nivel de activación o ansiedad estado es una variable que constantemente se ha relacionado con el rendimiento en deporte, encontrándose como los síntomas cognitivos y somáticos afectan negativamente al rendimiento (Hanin, 1997). Es esperable que en música ocurra algo similar o incluso que su efecto sea más acentuado al tratarse de una actividad motórica fina que requiere el movimiento específico, planeado y perfectamente intencionado de segmentos muy concretos del cuerpo, como son los dedos. De este modo, síntomas somáticos como por ejemplo el temblor ocasionado por la ansiedad estado afectará de forma directa en el toque del instrumento. Se puede esperar que si esto ocurre de forma frecuente, el estudiante tienda a evitar situaciones de evaluación convirtiéndose potencialmente el conservatorio y la música en una actividad poco recompensante; explicándose así la relación encontrada entre la variable competencia percibida y la intención de abandonar.

Por otro lado, la edad es un factor que, como se ha visto, influye en variables psicológicas relevantes para el rendimiento. Así, se conoce que las personas de mayor edad experimentan mayor estrés y afirman tener menos tiempo para estudiar que los más pequeños de la muestra. En danza se encontró como la competencia percibida diminuía conforme se aumentaba de curso (García-Dantas y Caracuel, 2011) pero en música, la relación significativa no es con el curso escolar sino con la edad de los estudiantes. Esta diferencia se puede explicar debido a que la variabilidad de edades en música es significativamente mayor que en danza. De este modo, mientras que las edades en una clase de danza suelen ser más o menos homogéneas ya que la edad en sí es un criterio para el acceso a estas enseñanzas, en música no ocurre lo mismo y la variedad de edades puede significar que en una misma clase existan personas de 14 y de 40 años.

Por último, no se puede olvidar las variables que mayor relación mantienen con el abandono. Se ha encontrado que las personas que presentan deseos de abandonar las enseñanzas de música son las que menor puntuación presentan en motivación básica, motivación cotidiana, competencia percibida y estrés. Del mismo modo que ocurría con los bailarines del estudio anteriormente citado, el apoyo que perciben de los padres, el nivel de activación que presentan previo a la actuación y el clima de clase no influyen de forma significativa en los deseos de abandonar. No obstante existe una diferencia que es importante resaltar; para los bailarines el apoyo que perciben del profesorado posee una gran influencia en el abandono, hecho que no sucede con tanta intensidad en los músicos (García-Dantas y Caracuel, 2011; Caracuel, GarcíaDantas y González, 2011).

Salvo las excepciones anteriormente citadas, todos los factores correlacionan significativamente con la probabilidad de abandonar; por ello se puede afirmar que niveles altos de motivación intrínseca (López de la Llave y Pérez-Llantada, 2006), competencia percibida (Taylor y Taylor, 1995) y estrés juegan un papel esencial en la continuidad del aprendiz de música en el Conservatorio.

Además, estos resultados confirman las indicaciones de Caracuel (2002), cuando se refería al valor que presentaba la autoconfianza o la creencia en la habilidad personal para la continuación de los deportistas en la actividad. Por otra parte, como manifiestan Taylor y Taylor (1995), bajos niveles de competencia en el intérprete favorecen el desencadenamiento de un círculo vicioso que implica un descenso de la confianza al actuar adecuadamente, lo que conlleva a su vez una disminución real en el rendimiento que favorece ese tipo de pensamientos negativos sobre las propias capacidades.

Entre las limitaciones que se han podido encontrar y las sugerencias para futuros estudios están la ampliación de la muestra abarcando diferentes conservatorios de España, así como, aumentando los cursos objeto de estudio. Sería bastante interesante conocer si esas relaciones se mantienen con el paso del tiempo o en cambio, son más específicas de los cursos estudiados.

Para terminar, investigaciones y experiencias aplicadas de la psicología y pedagogía tendrían mucho que aportar en este terreno, ya no sólo para mejorar la adherencia a la actividad, sino lo que es más importante, para mejorar la calidad de vida del alumnado y el profesorado que se encuentra diariamente en los conservatorios. Como se dijo anteriormente, abandonar o interrumpir la actividad no tiene por qué ir ligado a consecuencias negativas, pero de forma habitual el cese del aprendizaje de la música a este nivel puede ir ligado a experiencias más o menos traumáticas. Gracias a este estudio se espera que si se reducen los niveles desadaptativos de estrés y se incrementa la motivación intrínseca y la competencia percibida el alumnado presentará menor probabilidad de abandonar las enseńanzas y por consiguiente mayor adherencia y disfrute en el Conservatorio Profesional de Música. 


\section{Referencias}

1. Bandura, A. (1997). Self-efficacy: The exercise of Control. New York: Freeman.

2. Caracuel, J.C. (2002). Motivación en el deporte y el ejercicio. En M. Hernández (ed.) Motivación animal y humana, 277-292. México: El Manual Moderno.

3. Caracuel, J.C., García-Dantas, A. y González, J. (2011). Dance and music students: common and different psychological characteristics. Actas $13^{\text {th }}$ FEPSAC European Congress of Sport Psychology. Madeira: Portugal

4. Carlin, M., Salguero, A., Márquez, S. y Garcés, E. (2009). Análisis de los motivos de retirada de la práctica deportiva y su relación con la orientación motivacional en deportistas universitarios. Cuadernos de Psicología del Deporte, 9,1, 85-99.

5. García-Dantas, A. y Caracuel, J.C. (2010). Competencia percibida en jóvenes bailarines: un estudio en el Conservatorio Profesional de Danza de Sevilla. Comunicación oral presentada en el XII Congreso Nacional de Psicología del Deporte y $1^{\circ}$ Jornada Internacional de la AMPDA. Universidad Autónoma de Madrid, España.

6. García-Dantas, A. y Caracuel J.C. (2011). Factores que influyen en el abandono en los conservatorios de danza. Revista Iberoamericana de Psicología del Ejercicio y del Deporte, 6, 79- 97.

7. García-Dantas, A., Caracuel, J.C., y Peñaloza, R. (2013). Intervención Formativa con el Profesorado de Danza e Influencia Motivacional en su Alumnado. Cuadernos de Psicología del Deporte, 13,2, 9-20.

8. González, M. D. y Torregrosa, M. (2009). Análisis de la retirada de la competición de élite: antecedentes, transición y consecuencias. Revista Iberoamericana de Psicología del Ejercicio y el Deporte, 4,1, 93-104.

9. Guillén, F. y Bara, M. (2009) Motivos do abandono precoce no esporte competitivo: un estudo prospectivo. Revista Mineira de Educação Física, 17,1, 21-37.

10. Hanin, Y.L. (1997): Emotions and athletic performance: Individual zones of optimal functioning model, European Yearbook of Sport Psychology, 1, 29-72.

11. López De La Llave, A. y Pérez-Llantada, M.C. (2006). Psicología para Intérpretes Artísticos. Madrid, España: Thomson.

12. Marquez, D. X.; Jerome, G. J., Mcauley, E., Snook, E. M. y Canaklisova, S. (2002). Self-efficacy manipulation and state anxiety responses to exercise in low active women. Psychology and Health, 17, 6, 783-791.

13. Martens, R., Vealey, R. y Burton, D. (1990). Competitive Anxiety in Sport. Champaing, IL: Human Kinetics.

14. Martin, J. J. y Gill, D. L. (1991). The relationship among competitive orientations, sport-confidence, self-efficacy, anxiety and performance. Journal of Sport and Exercise Psychology, 13, 149-159.

15. Mérida, A. y Caracuel, J.C., (2001). Cuestionario sobre factores de inicio mantenimiento y abandono del deportelactividad física. Universidad de
Sevilla. Informe no publicado.

16. North, A., Colley, A. y Hargreaves, D. (2003). Adolescents' perceptions of the music of male and female composers. In E. Wehr-Flowers (2006). Differences between Male and Female Students' Confidence, Anxiety, and Attitude toward Learning Jazz Improvisation. Journal of Research in $\mathrm{Mu}-$ sic Education, 54, 4, 337-349.

17. Pajares, F. (1996). Self-efficacy beliefs in achievement settings. Review of Educational Research, 66, 543-578.

18. Quested, E., Bosch, J. A., Burns, V. E., Cumming, J., Ntoumanis, N. y Duda J. L. (2011). Basic Psychological Need Satisfaction, Stress-Related Appraisals, and Dancers' Cortisol and Anxiety Responses. Journal of Sport and Exercise Psychology, 33, 828-846.

19. Sanna M. Nordin-Bates, S. M., Cummin, J., Aways, D. y Sharp, L. (2011). Imagining Yourself Dancing to Perfection? Correlates of Perfectionism Among Ballet and Contemporary Dancers. Journal of Clinical Sport Psychology, 5, 58-76.

20. Scanlan, T. (2006) The Sport Commitment Model. Journal of Sport and Exercise Psychology. 15(1), 1-15.

21. Solmon, M., Lee, A. y Belcher, D. (2003). Beliefs about gender appropriateness, ability, and competence in physical activity. In E. Wehr-Flowers (2006). Differences between Male and Female Students' Confidence, Anxiety, and Attitude toward Learning Jazz Improvisation. Journal of Research in Music Education, 54, 4, 337-349.

22. Tavani, C. y Losh, S. (2003). Motivation, self-confidence, and expectations as predictors of the academic performances among our high school students. In E. Wehr-Flowers (2006). Differences between Male and Female Students' Confidence, Anxiety, and Attitude toward Learning Jazz Improvisation. Journal of Research in Music Education, 54, 4, 337 349.

23. Taylor, J. y Taylor, C. (2008). Psicología de la danza. Madrid, España: Gaia.

24. Vermeer, H., Boekaerts, M. \& Seegers, G. (2000). Motivational and gender differences: Sixth-grade students' mathematical problem-solving behavior. In E. Wehr-Flowers (2006). Differences between Male and Female Students' Confidence, Anxiety, and Attitude toward Learning Jazz Improvisation. Journal of Research in Music Education, 54, 4, $337-$ 349.

25. Walker, I. J. y Nordin-Bates, S. M. (2010). Performance Anxiety Experiences of Professional. Ballet Dancers. The Importance of Control. Journal of Dance Medicine and Science, 14, 4,133-145.

26. Wehr-Flowers, E. (2006). Differences between male and female students' confidence, anxiety, and attitude toward learning jazz improvisation. Journal of Research in Music Education, 54, 4, 337-349. 